\title{
Joint resource allocations for remote radio head deployments with coherent transmitter
}

Tan Wang ${ }^{1,2^{*}}$, Ying Wang ${ }^{1,2}$, Cong Shi ${ }^{1,2}$ and Ping Zhang ${ }^{1,2}$

\begin{abstract}
To deal with the future high demands of wireless traffic and energy consumption, one type of heterogeneous deployments is to distribute the base station antennas throughout the entire cell, which is called remote radio head $(\mathrm{RRH})$. In view of radio resource management, the joint resource allocation in broadband orthogonal frequency division multiple access RRH (OFDMA-RRH) has not been thoroughly studied, which includes the antenna port selection, subcarrier assignment and power allocation under the power constraints per antenna port. This article focuses on the downlink resource allocation in OFDMA-RRH, where the coherent transmitter with phase steering is used. The problem to maximize the system spectrum efficiency (SE) is formulated, and solved by a dual decomposition method, where the decomposed sub-problems in the dual domain are solved by analytic geometry. In the optimal solution, the system power is always exhaustively used. As another contribution, this article further proposes that in the optimal solution, there is a considerable part of system power which makes little contribution to the SE and thus should be saved. Specifically, the energy efficiency is analyzed for an individual subcarrier transmission. A proportional power allocation (PPA) method is proposed to achieve a local optimality for each subcarrier. By applying the PPA to every subcarrier in order to maximize the system SE, a new resource allocation problem is formulated and solved. Discussions are also presented for the proposed algorithms on the computational complexity and feasibility in multi-cell cases. Simulation results show that the optimal algorithm consumes all the system power and has good convergence. The algorithm applying PPA can effectively improves the system power efficiency while achieving the SE optimality.
\end{abstract}

\section{Introduction}

The last decade has witnessed the transition of multipleinput multiple-output (MIMO) and orthogonal frequency division multiple access (OFDMA) from theoretical concepts to practical technologies for enhancing performance of wireless networks. The dynamic subcarrier assignment (SA), adaptive power allocation (PA) and multiple spatial channels significantly improve the system capacity $[1,2]$. As their standardization work in the 3rd generation partnership project (3GPP) and other organizations approaching to an end, challenges are raised by the new round of high demands of wireless traffic, which is spurring new radio technologies, deployment infrastructures and radio resource allocation methods to be studied. Meanwhile, the Information \& Communication Technology (ICT) industries are facing an increase in

\footnotetext{
* Correspondence: wangtan0281@gmail.com

'Key Laboratory of Universal Wireless Communication, Ministry of Education, Beijing, China

Full list of author information is available at the end of the article
}

associated energy consumption of $16-20 \%$ per year [3], which highlights the urgent desire for researches focusing on energy efficiency (EE).

Due to the limited power, the wireless transmission distance cannot be too large if the data rate is very high. Improved and new system architectures are being investigated. One such architecture is to distribute the base station (BS) antennas throughout the entire cell via optical fiber. It is called remote radio head (RRH), specified as one type of heterogeneous deployments in 3GPP [4], or distributed antenna systems (DAS) [5]. By shortening the transmission distance, the RRH system has an inherent advantage in power saving. It could also benefit from macro diversity and signal combining from distributed RRH ports. Typically, a RRH port is equipped with only a transceiver, which is low-cost and easy to be deployed. From the view of radio resource management, combining OFDMA with RRH can provide more system performance gain by exploiting diversity in both the frequency and space domains. In OFDMA-RRH, to maximize the 
system spectrum efficiency (SE), the BS should jointly manage the SA, PA and together the RRH antenna port selection. Moreover, the power constraints per port (PCPP) should be taken into account.

\subsection{Related study}

Research interests in RRH have grown considerably over the last few years. The topics include from capacity [6,7], joint processing [8-10], location optimization [11] to those considering the practical imperfect conditions such as backhaul constraints [12] and asynchronous interference [13].

However, the literatures of joint resource allocation optimization in downlink OFDMA-RRH are rather limited. Many insightful researches focus on the optimal PA for single-band multi-user downlink transmission with PCPP $[9,10,14,15]$, where OFDMA is not considered. In [16], the joint problem is decomposed into two sub-problems: antenna-level and user-level scheduling. The RRH port selection is included in the antenna-level scheduling. Similar schemes can be found in [17], where a hierarchical algorithm is proposed for wireless mesh networks. In these study, however, each user connects with only one RRH port, which simplifies the PCPP. In [8], an improved null steering downlink OFDMA-RRH system is proposed which reduces both the inter-user correlation and the near-far problem resulting in a significant enhancement, but the PA therein is not jointly optimal. Our previous study in [18] addresses the joint problem to maximize system SE, but the assumption is made that subcarriers are assigned by the round robin method. The author of [19] additionally considers the minimum data rate as fairness constraint by setting a predefined minimum number of assigned subcarriers to each user. Nevertheless, the equal power allocation is used in his network flow approach. In a word, the joint resource allocation optimization in OFDMA-RRH has not been thoroughly studied.

A tradeoff between SE and EE is proposed in [20], where the user's utility as a function of transmit power is proposed. Among the previous study for energy-saving in resource allocation, some researchers focus on how to minimize transmit power under constraints such as user signal-to-interference-plus-noise ratio (SINR) threshold [15]. Some studies are trying to optimize the energy efficiency [21]. However, most prior work in this area has concentrated on traditional MIMO-OFDM systems. Recently some literatures for energy-efficient techniques are related to denser deployment of BSs $[22,23]$. But the BSs in these studies will interfere with each other and do not cooperate as in RRH. Therefore, the research on energy-saving resource allocation in OFDMA-RRH is still worth exploring.

\subsection{Contribution}

This article is dedicated to the joint downlink resource allocation in OFDMA-RRH, which includes the optimization of SA, PA, and RRH port selection. The coherent transmitter with phase steering is used in the system model, as the necessary method to maximize receiving signal-to-noise-ratio (SNR) under PCPP. The resource allocation problem to maximize the system SE is formulated. The dual decomposition method is used for obtaining its solution, where the decomposed sub-problems in the dual domain are solved from the perspective of analytic geometry.

In the optimal solution, the system power is always exhaustively used. As an innovation, this article proposes that, in the optimal solution, not all the power is used in an efficient way. For power-saving while achieving the optimal SE, the EE is analyzed for an individual subcarrier transmission. A proportional PA (PPA) method is proposed to achieve a local optimality for each subcarrier. Then by applying the PPA to every subcarrier for maximizing the system SE, a new resource allocation problem is formulated and solved, which is trying to spend the system power more efficiently. Next the computational complexity are compared and the feasibility in multi-cell cases are analyzed. Simulation results show that the optimal algorithm consumes all the system power and has good convergence. The algorithm applying PPA can save a considerable part of system power, thanks to the distributed feature in RRH deployments.

The reminder of this article is organized as follows: Section 2 describes the common downlink system model in OFDMA-RRH. In Section 3, the optimal resource allocation problem is formulated and solved. The EE analysis, PPA method and new problem are presented in Section 4. Discussion is made in Section 5 on the computational complexity and multi-cell feasibility. Section 6 gives the simulation results. We conclude the article in Section 7.

\section{System model}

Considering the downlink transmission, the BS has $M$ single-antenna RRH ports distributed in the whole cell area. Perfect wireline connection between BS and each $\mathrm{RRH}$ port is assumed, i.e., no backhaul constraints and transmission delays. The BS is regarded as a control center for the system. There are $K$ users uniformly distributed in the area. The frequency bandwidth is divided into $N$ subcarriers, which are the minimum resource units for SA. Denote $l_{k}[m, n]$ and $h_{k}[m, n]$ as the largescale fading and channel impulse response from $R R H$ port $m$ to user $k$ on subcarrier $n$, respectively.

During each scheduling period, it is assumed that the channels have flat fading and each subcarrier can be assigned to only one user. Denote $\rho_{k}[n]$ as the SA 
indicator. $\rho_{k}[n]=1$ only if subcarrier $n$ is assigned to user $k$. Otherwise $\rho_{k}[n]=0$. It satisfies that $\sum_{k=1}^{K} \rho_{k}[n]=1, \forall n$.

The system arranges a set of RRH ports, $\mathcal{M}_{k}[n]$, for the transmission of subcarrier $n$ assigned to user $k$. The RRH ports in each $\mathcal{M}_{k}[n]$ form a macro MIMO transmitter. The BS is assumed to have the perfect channel state information (CSI). This allows some transmission strategies to be used, such as transmit maximum ratio combining. But the PCPP must be satisfied in designing the transmit weight vector.

The sum power of the system is denoted as $P_{T}$. For presentation simplicity, it is assumed that each $\mathrm{RRH}$ port has the same power limit, denoted as $P, P=\frac{P_{T}}{M}$. In fact, this will not reduce the difficulty of the problem in the following sections. Let $p_{k}[m, n]$ be the transmit power of RRH port $m$ for subcarrier $n$, then the PCPP could be expressed as:

$$
\sum_{k=1}^{K} \sum_{n=1}^{N} \rho_{k}[n] p_{k}[m, n] \leq P, p_{k}[m, n] \geq 0, \forall m
$$

Note that at first it is supposed all the RRH ports are used in the transmission for each subcarrier, i.e., $\mathcal{M}_{k}[n]=\{1,2, \ldots, M\}, \forall n$. If $\rho_{k}[n]=1$ and $p_{k}[m, n]=0$, RRH port $m$ will not allocate any power for subcarrier $n$ assigned to user $k$, i.e., $m \notin \mathcal{M}_{k}[n]$, which means the RRH port selection is implicitly indicated by SA and PA results.

\subsection{Independent weight vector}

If it is assumed that subcarrier $n$ is assigned to user $k$, the related receiving signal, which is transmitted from each RRH port after being weighted, will be:

$$
y_{k}[n]=\boldsymbol{h}_{k}[n] \boldsymbol{P}_{k}[n] \boldsymbol{\omega}_{k}[n] x_{k}[n]+z_{k}[n],
$$

where

$$
\begin{aligned}
& h_{k}[n]=\left(\sqrt{l_{k}[1, n]} h_{k}[1, n], \ldots, \sqrt{l_{k}[M, n]} h_{k}[M, n]\right), \\
& \boldsymbol{P}_{k}[n]=\operatorname{diag}\left(\sqrt{p_{k}[1, n]}, \sqrt{p_{k}[2, n]}, \ldots, \sqrt{p_{k}[M, n]}\right),
\end{aligned}
$$

$x_{k}[n]$ is the unitary transmitted symbol for user $k, z_{k}$ $[n]$ is the additive Gaussian noise with variance $\sigma^{2}$ and $\omega_{k}[n]$ is the transmit weight vector.

To guarantee the PCPP, the coherent transmitter is applied. As proved in [7], the optimal weight vector, which is necessary to maximize the receiving SNR, is obtained by phase steering:

$$
\omega_{k}[n]=\left(\frac{h_{k}[1, n]^{H}}{\left|h_{k}[1, n]\right|^{1}}, \ldots, \frac{h_{k}[M, n]^{H}}{\left|h_{k}[M, n]\right|}\right)^{T} .
$$

Note that the normalized operation is done independently by each RRH port, to accommodate the channel impulse response. Then the receiving SNR will be:

$$
\gamma_{k}[n]=\frac{1}{\sigma^{2}}\left(\sum_{m=1}^{M} \sqrt{G_{k}[m, n] p_{k}[m, n]}\right)^{2} .
$$

where $G_{k}[m, n]=l_{k}[m, n]\left|h_{k}[m, n]\right|^{2}$ is the combined channel gain, followed by the SE as $C_{k}[n]=\log _{2}\left(1+\gamma_{k}\right.$ $[n])$. The downlink transmission process of the system and the list of symbols used in this article are shown in Figure 1 and Table 1, respectively.

\section{Optimal resource allocation}

Traditionally, the optimization problem to maximize the system SE can be formulated as:

$$
\begin{aligned}
& \text { P1: } \max _{p, \rho} \sum_{k=1}^{K} \sum_{n=1}^{N} \rho_{k}[n] \cdot \log \left(1+\frac{1}{\sigma^{2}}\left(\sum_{m=1}^{M} \sqrt{G_{k}[m, n] p_{k}[m, n]}\right)^{2}\right), \\
& \text { s.t. } \quad \text { C1: } \quad \sum_{k=1}^{K} \sum_{n=1}^{N} \rho_{k}[n] p_{k}[m, n] \leq P, \forall m, \\
& \text { C2: } \quad \rho_{k}[n] \in\{0,1\}, \forall k, n, \quad \sum_{k=1}^{K} \rho_{k}[n]=1, \forall n, \\
& \text { C3: } \quad p_{k}[m, n] \geq 0, \forall k, m, n .
\end{aligned}
$$

P1 is not easy to be solved directly. According to [24], the duality gap is nearly zero when the number of subcarriers is sufficiently large in multiband systems. It is an effective way to obtain the solution of the original problem by solving the dual problem, where the dual decomposition method is suggested as in [24]. Therefore, this article uses this method to solve P1. The Lagrangian function of P1 is:

$$
\begin{aligned}
\mathcal{L}(\boldsymbol{p}, \boldsymbol{\rho}, \boldsymbol{\lambda})= & \sum_{k=1}^{K} \sum_{n=1}^{N} \rho_{k}[n] \cdot \log \left(1+\frac{1}{\sigma^{2}}\left(\sum_{m=1}^{M} \sqrt{G_{k}[m, n] p_{k}[m, n]}\right)^{2}\right)+ \\
& \sum_{j=1}^{M} \lambda_{j}\left(\sum_{k=1}^{K} \sum_{n=1}^{N} \rho_{k}[n] p_{k}[m, n]-P_{\prime}\right),
\end{aligned}
$$

where $\lambda=\left[\lambda_{1}, \ldots, \lambda_{M}\right]$ is the Lagrange multiplier corresponding to $\mathrm{C} 1$ in $\mathrm{P} 1$. Note that $\mathrm{C} 2$ and $\mathrm{C} 3$ are not taken into account in the Lagrangian function. However, it will be satisfied in the dual domain when solving the problem as shown in the following.

The Lagrangian dual function corresponding to P1 is formulated as follows:

$$
g(\lambda)=\max _{p, \rho} \mathcal{L}(p, \rho, \lambda)
$$

The dual optimization problem corresponding to $\mathrm{P} 1$ is given by:

$$
\begin{array}{ll}
\min _{\lambda} & g(\lambda) \\
\text { s.t. } & \lambda_{j} \leq 0, \forall j .
\end{array}
$$




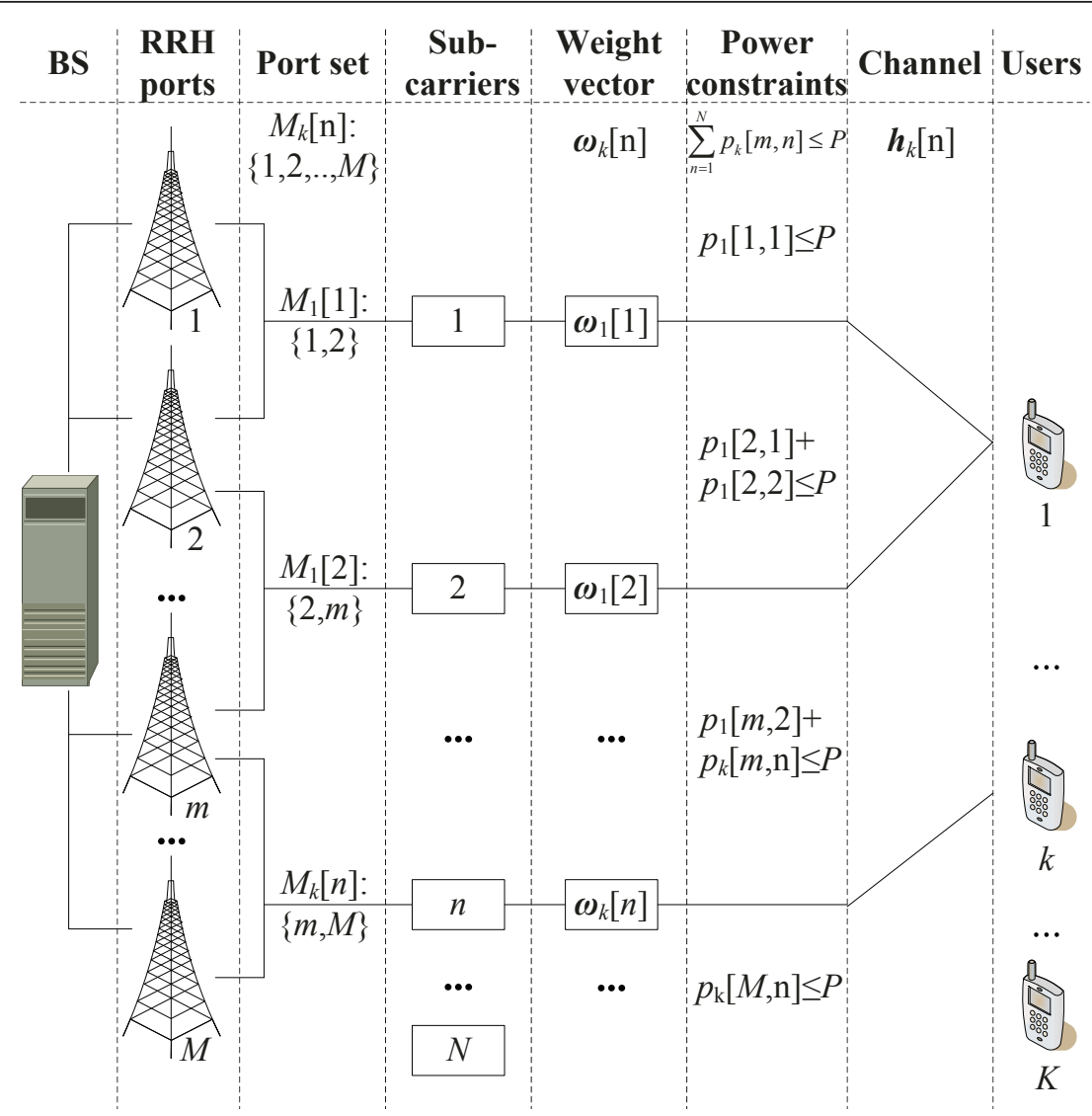

Figure 1 Downlink system transmission procedure. This figure goes with the system model, to make readers better understand the downlink system transmission procedure.

The Lagrangian function $\mathcal{L}(\boldsymbol{p}, \boldsymbol{\rho}, \boldsymbol{\lambda})$ is linear in $\lambda$ for fixed $\boldsymbol{p}$ and $\boldsymbol{\rho}$, and $g(\lambda)$ is the maximum one of these linear functions. Therefore, the dual optimization problem (10) is convex. The Lagrangian dual function can be decomposed into $N$ independent optimization problems, shown as:

$$
g(\boldsymbol{\lambda})=\sum_{n=1}^{N} \mathcal{J}_{n}(\boldsymbol{\lambda})-\sum_{j=1}^{M} \lambda_{j} \cdot P,
$$

where

$$
J_{n}(\lambda)=\max _{\rho, \rho}\left[\sum_{k=1}^{K} \rho_{k}[n] \cdot \log \left(1+\frac{1}{\sigma^{2}}\left(\sum_{m=1}^{M} \sqrt{G_{k}[m, n] p_{k}[m, n]}\right)^{2}\right)+\sum_{j=1}^{M} \sum_{k=1}^{K} \lambda_{j} \rho_{k l}\left[n p_{k k}[m, n]\right] .\right.
$$

Since each subcarrier can only be assigned to one user, Equation (12) actually indicates a rule for SA which is to search the user that maximizes Equation (12) for a specific subcarrier. For a given user $k$ and $\rho_{k}$ $[n]=1$, the optimal $P_{k}^{*}[m, n], \forall m$, can be obtained from solving P2 as follows:

$$
\text { P2 : } \max _{p} \log \left(1+\frac{1}{\sigma^{2}}\left(\sum_{m=1}^{M} \sqrt{G_{k}[m, n] p_{k}[m, n]}\right)^{2}\right)+\sum_{j=1}^{M} \lambda_{j} p_{k}[m, n] .
$$

As for the solution of P1, each subcarrier is assigned to the corresponding user according to Equation (12) and then update the Lagrangian multipliers. The iterative process is continuing until convergence. The solution of P2 is given in Lemma 1.

Lemma 1. For fixed $\lambda$, the solution of $\mathrm{P} 2$ is:

$$
p_{k}^{*}[m, n]=\left[\frac{B-\ln 2}{B^{2} \ln 2} \cdot \frac{G_{k}[m, n]}{\sigma^{2} \lambda_{m}^{2}}\right]^{+},
$$

where $[x]^{+}=\max \{x, 0\}$ and $B=\sum_{m=1}^{M} \frac{G_{k}[m, n]}{-\sigma^{2} \lambda_{m}}$.

Proof. From the perspective of analytic geometry, the proof is presented in Appendix 1. Now the optimal PA can be obtained given $\lambda$, which is denoted as $\boldsymbol{p}^{*}(\boldsymbol{\lambda})$. Substituting it into Equation (12), we can yield $\mathcal{J}_{n}(\lambda)$. In order to minimize the dual function, the sub-gradient method is adopted in this article [25], to update the Lagrangian multipliers and guarantee the convergence to the global optimum. The sub-gradient of $g(\lambda)$ is calculated according to Lemma 2. 
Table 1 List of the notations used throughout this article

\begin{tabular}{ll}
\hline Notations & Explanation \\
\hline$M$ & The total RRH port number \\
$K$ & The total user number \\
$N$ & The total subcarrier number \\
$W$ & The system bandwidth \\
$m_{,} n, k$ & The index of RRH port, subcarrier and user, respectively \\
$I_{k}[m, n]$ & The large-scale channel fading \\
$h_{k}[m, n]$ & The channel impulse response \\
$\rho_{k}[n]$ & The subcarrier assignment indicator \\
$\mathcal{M}{ }_{k}[n]$ & A set of RRH ports for the transmission of a subcarrier \\
$P_{T}$ & The total system power \\
$P$ & The power limit of each RRH port \\
$y_{k}[n]$ & The receiving signal \\
$x_{k}[n]$ & The unitary transmitted symbol \\
$z_{k}[n]$ & The additive Gaussian noise \\
$N_{0}$ & The noise power \\
$\omega_{k}[n]$ & The transmit weight vector \\
$\gamma_{k}[n]$ & The receiving SNR \\
$G_{k}[m, n]$ & The combined channel gain \\
$C_{k}[n]$ & The spectrum efficiency \\
$\mathcal{L}(\cdot)$ & The Lagrangian function \\
$g(\cdot)$ & The Lagrangian dual function \\
$\alpha, \beta, \lambda$ & The Lagrangian multipliers \\
$\Delta \alpha_{1} \Delta \beta, \Delta \lambda$ & The sub-gradients of Lagrangian multipliers \\
$\mathcal{J}(\cdot)$ & The dual decomposition function \\
$\eta$ & The energy efficiency \\
$\mu_{k}[n]$ & The power-scaling coefficient \\
\hline &
\end{tabular}

Lemma 2. The sub-gradient of $g(\lambda)$ is:

$$
\Delta \lambda_{j}=\sum_{k=1}^{K} \sum_{n=1}^{N} \rho_{k}^{*}[n](\lambda) p_{k}^{*}[m, n](\lambda)-P, j=1,2, \ldots, M .
$$

Proof. To be presented in Appendix 2.

According to Lemma 2, the update of Lagrangian multipliers are designed to be:

$$
\lambda_{j}^{l+1}=\left[\lambda_{j}^{l}-s_{\lambda_{j}}^{l}\left(\sum_{k=1}^{K} \sum_{n=1}^{N} \rho_{k}^{*}[n](\lambda) p_{k}^{*}[m, n](\lambda)-P\right)\right]^{+}, \forall j
$$

where $l$ is the iteration index, $s_{\lambda_{j}}^{l}$ is the appropriate positive step-size sequence. After yielding the optimal Lagrangian multipliers, denoted as $\lambda^{*}$, the optimal $\boldsymbol{p}^{*}$ is obtained by substituting $\lambda^{*}$ into Equation (14). Then by combining with Equation (12), the optimal SA is indicated. The pseudocode of the proposed algorithm for P1 is described in Algorithm 1, where $\varphi$ represents the empty set.

\section{Power-saving resource allocation}

In the optimal solution, the system power is always exhaustively used. However, from the analysis in this section, it is shown that there will be a part of power which is used in a low efficiency. Then a new problem is formulated, to find out in which manner the power is allocated efficiently.

\section{Algorithm 1 Optimal resource allocation.}

1: Subcarrier set: $\mathcal{N}=\{1,2, \ldots, N\}$, User set: $\mathcal{K}=\{1,2, \ldots, K\}$.

\section{2: Calculate optimal Lagrangian multipliers}

3: Initialization: $\lambda^{0}$

4: while stoping criterion is not satisfied do

5: (1) Compute $p_{k}^{*}[m, n]\left(\lambda^{0}\right)$ according to Equation

6: (2) Compute $\mathcal{J}_{n}\left(\lambda^{0}\right), \forall n \in \mathcal{N}$ according to Equation (12);

7: $\quad$ (3) Compute $g\left(\lambda^{0}\right)$ according to Equation (11);

8: (4) Update $\lambda^{0}$ according to Equation (16);

9: end while

10: Joint resource allocation

11: while $\mathcal{N} \neq \phi$ do

12: (1) Compute optimal $p_{k}^{*}[m, n]\left(\lambda^{*}\right), \forall n \in \mathcal{N}, k \in \mathcal{K}$, according to Equation (14);

13: (2) Find a pair of $\left(n^{*}, k^{*}\right)$ which maximizes Equation (12);

14: (3) Assign subcarrier $n^{*}$ to user $k^{*}$, and update $\rho_{k^{*}}$ $\left[n^{*}\right]=1$

15: (4) Set $p_{k}[m, n]=p_{k}^{*}[m, n]\left(\lambda^{*}\right), k=k^{*}$ and $p_{k}[m$, $n]=0, \forall k \neq k^{*}$

16: $\quad$ (5) $\mathcal{N}=\mathcal{N}-\left\{n^{*}\right\}$

17: end while

\subsection{EE function}

Defined in [26], the EE function is shown to be:

$$
\eta(P)=\frac{W}{P} \log _{2}\left(1+\frac{c P}{E N_{0}}\right),
$$

where $W$ represents the bandwidth, $P$ is the transmit power, $c$ is the channel gain and $N_{0}$ denotes the noise power. This article mainly explores the relationship between $P$ and $\eta(P)$. In fact, in our study, $W$ represents an individual subcarrier, the bandwidth of which is fixed. The channel gain $c$ can also be regarded as a constant in each channel realization.

Define $f(x)=\frac{1}{x} \log (1+w x), w>0$, we have the following lemma.

Lemma 3. Function $f(x)=\frac{1}{x} \log (1+w x), w>0$ is monotonically decreasing when $x>0$.

Proof. To be presented in Appendix 3.

From Lemma 3 and $\lim _{x \rightarrow+\infty} f(x)=0$ and $\lim _{x \rightarrow+\infty} f(x)=\frac{w}{\ln 2}$, it is shown that $\eta$ approaches zero when $P$ tends to infinity, and converges to $\frac{w}{\ln 2}$ when $P$ 
approaches zero. However, in practical systems, the calculation of EE is complicated. It is not only closely related to the transmission distance, channel model and noise figure, but also determined by the circuit power. It is not reasonable to make the transmit power to be a small value to gain a high EE, due to the circuit power [26]. In this context, this article focuses on how to save power while approaching the SE optimality.

\subsection{Individual subcarrier transmission in RRH}

Firstly the case of individual subcarrier transmission is studied to find out that, given a total transmit power limit, how to maximize its receiving SNR. In this section, a power-scaling coefficient is defined for each subcarrier, denoted as $\mu_{k}[n]$. The amount of power allocated to subcarrier $n$ is $\sum_{k=1}^{K} \rho_{k}[n] \mu_{k}[n] P_{T}$. It satisfies that

$$
\sum_{k=1}^{K} \sum_{n=1}^{N} \rho_{k}[n] \mu_{k}[n] \leq 1, \mu_{k}[n] \geq 0, \forall n
$$

The following lemma is adopted to acquire the PA result and receiving SNR.

Lemma 4. There are $M$ RRH ports, subcarrier $n$ is assigned to user $k$, and the sum power allocated to this subcarrier is $\mu_{k}[n] P_{T}$, i.e., $\sum_{m=1}^{M} p_{k}[m, n]=\mu_{k}[n] P_{T}$. To maximize the receiving SNR, the transmit power of $\mathrm{RRH}$ port $m$ is (when the PCPP is not considered):

$$
p_{k}^{*}[m, n]=\frac{G_{k}[m, n]}{\sum_{m=1}^{M} G_{k}[m, n]} \cdot \mu_{k}[n] P_{T}, \quad m=1,2, \ldots, M,
$$

and the maximum SNR is $\frac{1}{\sigma^{2}} \mu_{k}[n] P_{T} \cdot \sum_{m=1}^{M} G_{k}[m, n]$.

Proof. To be presented in Appendix 4.

Lemma 4 shows that, to maximize the SNR for subcarrier $n$, the optimal amount of power used by each RRH, $p_{k}^{*}[m, n]$, is linearly dependent with the channel gain. We name this PA method for one subcarrier as proportional PA (PPA). Without loss of generality, it is further assumed:

$$
p_{k}^{*}[1, n] \geq p_{k}^{*}[2, n] \geq \cdots \geq p_{k}^{*}[M, n] .
$$

When considering PCPP, $p_{k}^{*}[1, n]$ can not be arbitrary large. If it is assumed that the maximum transmission power of RRH port 1 for subcarrier $n$ is $P_{0}$, we can yield the following lemma.

Lemma 5. Let $p_{k}^{*}[n]=\left(p_{k}^{*}[1, n], \ldots, p_{k}^{*}[M, n]\right)$ and assume $p_{k}^{*}[1, n] \geq p_{k}^{*}[2, n] \geq \cdots \geq p_{k}^{*}[M, n]$, the following PA adjustments will lead to changes in EEas:
1. When $p_{k}^{*}[1, n]<P_{0}$, linearly increasing $\boldsymbol{p}_{k}^{*}[n]$ to $\boldsymbol{p}_{k}^{\prime}[n]$, where $p_{k}^{*}[1, n] \leq p_{k}^{\prime}[1, n] \leq P_{0}$, will bring down the EE.

2. When $p_{k}^{*}[1, n]=P_{0}$, increasing any of $p_{k}^{*}[i, n], i=2, \ldots, M$ will bring down the EE.

3 . When $p_{k}^{*}[1, n]>P_{0}$, linearly reducing $\boldsymbol{p}_{k}^{*}[n]$ to $\boldsymbol{p}_{k}^{\prime \prime}[n]$, where $p_{k}^{\prime \prime}[1, n] \leq P_{0}$, will improve the EE.

Proof. To be presented in Appendix 5.

Lemma 5 shows that besides the power allocated by PPA, additional allocation will result in lower efficiency. It also indicates that decreasing allocated power may improve EE. However, achieving the optimal EE is not the concentration in the scope of this article. For intuitive understanding, Figures 2 and 3 show an example to demonstrate Lemma 5.2. There is only one user $(k=1)$ and one subcarrier $(n=1)$ but with different RRH port numbers. The positions of RRH ports are regularly, as shown in Figure 4. For details of simulation configurations, please refer to Table 2 .

Two mthods are compared. One is the proposed PPA, and make sure that port $i=\arg \max _{m} p_{1}[m, 1]$ achieves its maximum power. This indicates that other RRH ports only utilize part of their power capability. The other one is the full PA, in which every RRH port uses its maximum power in the transmission.

It is shown in Figure 2 that full PA indeed improves the SNR by the exhaustive use of power of every RRH port. The improvement in SE goes from $7.6 \%$ to $19.1 \%$ as the RRH port number increases. However, its EE is dramatically lower than that of PPA (For simplicity, the EE is obtained directly from SE divided by the transmit power).

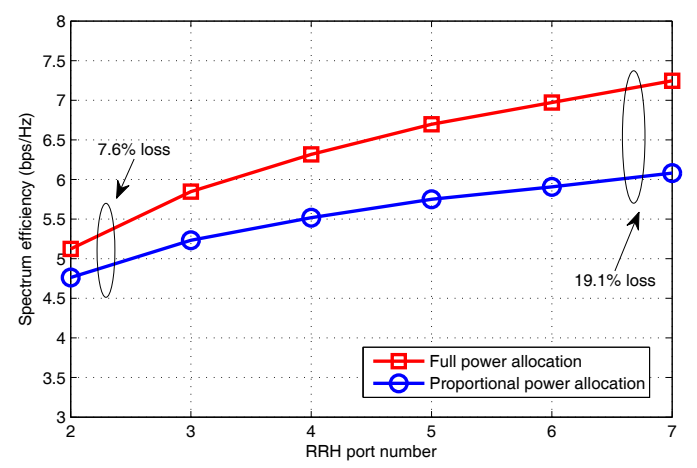

Figure 2 Comparisons of spectrum efficiency (SE) between proportional power allocation (PPA) and full power allocation. Figure 2 shows the spectrum efficiency (bps/Hz) of proportional power allocation (PPA) and full power allocation versus different RRH port numbers. Legend of curves from top to bottom: Full power allocation; Proportional power allocation. 


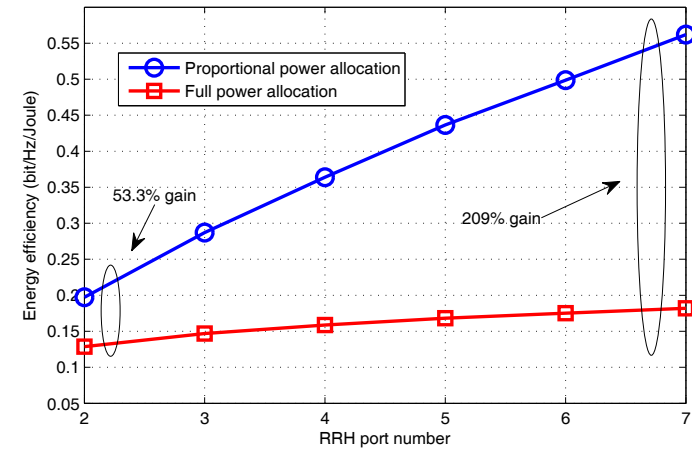

Figure 3 Comparisons of energy efficiency (EE) between proportional power allocation (PPA) and full power allocation. Figure 3 shows the energy efficiency (bit/Hz/Joule) of proportional power allocation (PPA) and full power allocation versus different RRH port numbers. Legend of curves from top to bottom: Proportional power allocation; Full power allocation.

The later method can use the system power more efficiently, with the gain of EE from $53.3 \%$ to $209 \%$.

\subsection{Power-saving problem}

The optimal solution of P1 will consume all the system power. On the other hand, the proportion in PA for each subcarrier can make efficient use of power. Based on these conclusions, this article proposes that, in the optimal solution, not all the power is used in an efficient way. An intuitive idea is formed, to apply PPA for every subcarrier. The scaling coefficients $\mu_{k}[n]$ are used here, to adjust the power allocated to different subcarriers. For each subcarrier, the SNR can be directly obtained from Lemma 4 accordingly. The new problem can be formulated as:

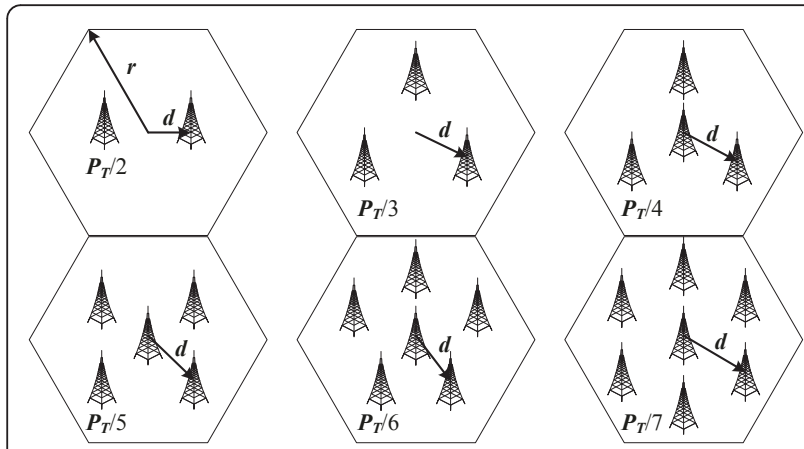

Figure 4 Simulation area with different numbers of RRH ports, $r=(500 / \sqrt{3}) \mathrm{m}, \boldsymbol{d}=(\mathbf{5 0 0 / 3}) \mathrm{m}$. Figure 4 shows the simulation area with 2-7 RRH ports in one macrocell, where

$r=(500 / \sqrt{3}) \mathrm{m}$ and $d=(500 / 3) \mathrm{m}$.
Table 2 Simulation parameters

\begin{tabular}{ll}
\hline Parameters & Assumption \\
\hline Scenario & 3GPP Case 1; resource block number, 50 \\
Cellular layout & Cell radius $(500 / \sqrt{3}) \mathrm{m}$; with 2-7 RRH ports \\
RRH port position & $(500 / 3) \mathrm{m}$ to the center (besides the central one) \\
UE distribution & 30 users in total, uniform distribution \\
Minimum & From users to RRH ports, $10 \mathrm{~m}$ \\
distance & \\
Tx power & $46 \mathrm{dBm}$ in total \\
Path loss & $\mathrm{L}=140.7+36.7 \log _{10}(R)$, for $2 \mathrm{GHz}$, R-distance in km \\
Shadowing & Standard deviation: $10 \mathrm{~dB}$ for RRH ports to users \\
Others & Penetration loss, $20 \mathrm{~dB}$; RRH/user antenna gain, \\
& $5 / 0 \mathrm{dBi}$
\end{tabular}

$$
\begin{aligned}
\text { P3: } \max _{\mu, \rho} \quad \sum_{k=1}^{K} \sum_{n=1}^{N} \rho_{k}[n] \cdot C_{k}[n], \\
\text { s.t. } \quad \text { C1: } \quad C_{k}[n]=\log \left(1+\frac{1}{\sigma^{2}} \mu_{k}[n] P_{T} \cdot \sum_{m=1}^{M} G_{k}[m, n]\right), \\
\text { C2 }: \sum_{k=1}^{K} \sum_{n=1}^{N} \rho_{k}[n] \mu_{k}[n] P_{T} \cdot \frac{G_{k}[m, n]}{\sum_{i=1}^{M} G_{k}[i, n]} \leq P, \forall m, \\
\text { C3 }: \sum_{k=1}^{K} \sum_{n=1}^{N} \rho_{k}[n] \mu_{k}[n] \leq 1, \\
\text { C4 }: \rho_{k}[n] \in\{0,1\}, \forall k, n, \sum_{k=1}^{K} \rho_{k}[n]=1, \forall n, \\
\text { C5 }: \mu_{k}[n] \geq 0, \forall k, n,
\end{aligned}
$$

where $\mathrm{C} 1$ and $\mathrm{C} 2$, respectively, represent the modified SE and PCPP. P3 can be solved using the dual decomposition method in the same way as P1, but the decomposed sub-problems in the dual domain are much easier. Due to the similarity. the procedure is briefly described. Denote $\sum_{m=1}^{M} G_{k}[m, n]=G_{k, n}$ for presentation simplicity and recall that $P_{T}=M P$. The Lagrangian function of P3 is:

$$
\begin{aligned}
\mathcal{L}(\boldsymbol{\mu}, \boldsymbol{\rho}, \boldsymbol{\alpha}, \beta)= & \sum_{k=1}^{K} \sum_{n=1}^{N} \rho_{k}[n] \cdot \log \left(1+\frac{1}{\sigma^{2}} \mu_{k}[n] P_{T} G_{k, n}\right)+ \\
& \sum_{j=1}^{M} \alpha_{j}\left(\sum_{k=1}^{K} \sum_{n=1}^{N} \rho_{k}[n] \mu_{k}[n] \cdot \frac{G_{k}[m, n]}{G_{k, n}}-\frac{1}{M}\right)+\beta\left(\sum_{k=1}^{K} \sum_{n=1}^{N} \rho_{k}[n] \mu_{k}[n]-1\right),
\end{aligned}
$$

where $\alpha=\left[\alpha_{1}, \ldots, \alpha_{M}\right], \beta$ are the Lagrange multipliers corresponding to $\mathrm{C} 2$ and $\mathrm{C} 3$ in P3. The Lagrangian dual function is formulated as:

$$
g(\boldsymbol{\alpha}, \beta)=\max _{\boldsymbol{\mu}, \boldsymbol{\rho}} \mathcal{L}(\boldsymbol{\mu}, \boldsymbol{\rho}, \boldsymbol{\alpha}, \beta)
$$

The dual optimization problem is then given by:

$$
\begin{aligned}
\min _{\boldsymbol{\alpha}, \beta} & g(\boldsymbol{\alpha}, \beta) \\
\text { s.t. } & \alpha_{j} \leq 0, \forall j, \quad \beta \leq 0,
\end{aligned}
$$

which is convex. Decompose the Lagrangian dual function into $N$ independent optimization problems as:

$$
g(\boldsymbol{\alpha}, \beta)=\sum_{n=1}^{N} \mathcal{H}_{n}(\boldsymbol{\alpha}, \beta)-\frac{1}{M} \sum_{j=1}^{M} \alpha_{j}-\beta,
$$


where

$$
\mathcal{H}_{n}(\boldsymbol{\alpha}, \beta)=\max _{\mu_{,}, \boldsymbol{\beta}}\left[\sum_{k=1}^{K} \rho_{k}[n] C_{k}[n]+\sum_{k=1}^{K} \sum_{j=1}^{M} \alpha_{j} \rho_{k}[n] \mu_{k}[n] \cdot \frac{G_{k}[m, n]}{G_{k, n}}+\beta \sum_{k=1}^{K} \rho_{k}[n] \mu_{k}[n]\right] .
$$

Define $\mu_{k}^{*}[n]$ as the optimal PA for given subcarrier $n$ if assigned to user $k$. For fixed $(\alpha, \beta)$, the $\mu_{k}^{*}[n]$ that maximizes the objective function in P3 can be directly obtained using Karush-Kuhn-Tucke (KKT) condition [25], which is:

$$
\left.\frac{\partial \mathcal{L}(\boldsymbol{\mu}, \boldsymbol{\rho}, \boldsymbol{\alpha}, \beta)}{\partial \mu_{k}[n]}\right|_{\mu_{k}[n]=\mu_{k}^{*}[n]}\left\{\begin{array}{l}
<0, \mu_{k}[n]=0, \\
=0, \mu_{k}[n]>0
\end{array} \quad \forall k, n .\right.
$$

Therefore, we have:

$$
\mu_{k}^{*}[n]=\left[-\frac{\sigma^{2}}{P_{T} G_{k, n}}-\frac{1}{T \ln 2}\right]^{+},
$$

where $T=\sum_{j=1}^{M} \alpha_{j} \cdot \frac{G_{k}[m, n]}{G_{k, n}}+\beta$. From Equation (28), we can get $\mathcal{H}_{n}(\boldsymbol{\alpha}, \beta)$.

Similar as Lemma 2 , the sub-gradient of $g(\alpha, \beta)$ is calculated as:

$$
\begin{aligned}
\Delta \alpha_{j} & =\sum_{k=1}^{K} \sum_{n=1}^{N} \rho_{k}^{*}[n](\boldsymbol{\alpha}, \beta) \mu_{k}^{*}[n](\boldsymbol{\alpha}, \beta) \cdot \frac{G_{k}[m, n]}{G_{k, n}}-\frac{1}{M} \\
\Delta \beta & =\sum_{k=1}^{K} \sum_{n=1}^{N} \rho_{k}^{*}[n](\boldsymbol{\alpha}, \beta) \mu_{k}^{*}[n](\boldsymbol{\alpha}, \beta)-1 .
\end{aligned}
$$

Accordingly, the update of Lagrangian multipliers are designed to be:

$$
\begin{aligned}
\alpha_{j}^{l+1} & =\left[\alpha_{j}^{l}-s_{\alpha_{j}}^{l}\left(\sum_{k=1}^{K} \sum_{n=1}^{N} \rho_{k}^{*}[n](\boldsymbol{\alpha}, \beta) \mu_{k}^{*}[n](\boldsymbol{\alpha}, \beta) \cdot \frac{G_{k}[m, n]}{G_{k, n}}-\frac{1}{M}\right)\right]^{+}, \forall j, \\
\beta^{l+1} & =\left[\beta^{l}-s_{\beta}^{l}\left(\sum_{k=1}^{K} \sum_{n=1}^{N} \rho_{k}^{*}[n](\boldsymbol{\alpha}, \beta) \mu_{k}^{*}[n](\boldsymbol{\alpha}, \beta)-1\right)\right]^{+},
\end{aligned}
$$

where $l$ is the iteration index, $s_{\alpha_{j}}^{l}$ and $s_{\beta}^{l}$ are the appropriate positive step-size sequences. The pseudocode of the proposed algorithm for P3 is described in Algorithm 2.

\section{Discussion}

In this section, the complexity analysis and the feasibility in multi-cell cases are discussed for the proposed algorithms.

\subsection{Complexity analysis}

Before the simulation results, to make fair comparisons, the computational complexity is analyzed for the proposed two algorithms. In the optimal resource allocation, the number

Algorithm 2 Resource allocation applying PPA.
1: Subcarrier set: $\mathcal{N}=\{1,2, \ldots, N\}$, User set: $\mathcal{K}=\{1,2, \ldots, K\}$.

2: Calculate optimal Lagrangian multipliers

3: Initialization: $\left(\alpha^{0}, \beta^{0}\right)$

4: while stoping criterion is not satisfied do

5: (1) Compute $\mu_{k}^{*}[n]\left(\alpha^{0}, \beta^{0}\right)$ according to Equation (28);

6: $\quad$ (2) Compute $\mathcal{H}_{n}\left(\boldsymbol{\alpha}^{0}, \beta^{0}\right), \forall n \in \mathcal{N}$ according to Equation (26);

7: (3) Compute $g\left(\alpha^{0}, \beta^{0}\right)$ according to Equation (25);

8: (4) Update $\left(\alpha^{0}, \beta^{0}\right)$ according to Equation (30); 9: end while

10: Joint resource allocation

11: while $\mathcal{N} \neq \phi$ do

12: (1) Compute optimal $\mu_{k}^{*}[n]\left(\boldsymbol{\alpha}^{*}, \beta^{*}\right), \forall n \in \mathcal{N}, k \in \mathcal{K}$, according to Equation (28);

13: (2) Find $k^{*}$ which maximizes Equation (26);

14: (3) Assign subcarrier $n^{*}$ to user $k^{*}$, and update $\rho_{k^{*}}\left[n^{*}\right]=1$;

15: (4) Set $p_{k}[m, n]$ according to Equation (19) for $k=k^{*}$ and $p_{k}[m, n]=0, \forall k \neq k^{*}$;

16: $\quad$ (5) $\mathcal{N}=\mathcal{N}-\left\{n^{*}\right\}$

17: end while

of iterations required to get the $\varepsilon$-optimal Lagrangian multipliers $\lambda^{*}$, i.e., $g(\lambda)-g\left(\lambda^{*}\right)<\epsilon$, is on the order of $O(1 /$ $\left.\varepsilon^{2}\right)$ [25]. In each iteration, the calculation of Equation (12) includes $K$ comparisons for each of $N$ subcarriers. Therefore, the total computational complexity of Algorithm 1 is $O\left(N K / \varepsilon^{2}\right)$. As for the power-saving resource allocation, the required number of iterations to get the $\varepsilon$ optimal $\left(\alpha^{*}, \beta^{*}\right)$ is also on the order of $O\left(1 / \varepsilon^{2}\right)$, followed by the same complexity in each iteration to calculate Equation (26) as $O(N K)$. Therefore, the computational complexity is on the same order, $O\left(N K / \varepsilon^{2}\right)$, for the two algorithms.

\subsection{Multi-cell cases}

As shown by the simulation results, the proposed algorithms are effective in single-cell case. For the multi-cell case, the distributed RRH ports from adjacent cells may change the interference statistics. However, from the theoretical point of view, a typical modeling of interference is to regard the noise plus interference as a complex Gaussian random variable [7]. In this context, the proposed algorithms can be taken as a basis, which is also applicable in multi-cell situations, as long as calculating different noise plus interference power $\sigma_{k}^{2}$ for each user. The variance is calculated jointly according to the largescale fading from interfering antenna ports and the noise power. By focusing on the single-cell case, we can 
actually obtain more insights into the PPA and better understand the power-saving feature of RRH, as shown in the following.

\section{Simulation results}

Consider a macrocell area and the deployment of RRH ports is illustrated in Figure 4. Each RRH port is with an omni-directional antenna. Other basic simulation parameters refer to 3GPP Long Term Evolution (LTE) heterogeneous deployments [4] as Table 2 shows. The minimum scheduling unit is a resource block, the total number of which is 50 . All the RRH ports have the same PCPP with a fixed total system power, i.e., $P=\frac{P_{T}}{M}$. Users with full buffer traffics are uniformly distributed in the cell. In the simulations, each illustrated point in the figure is obtained averaging the results of 100 user drops, each with 500 channel realizations.

Figure 5 shows the SE performances of Algorithms 1 and 2 versus different RRH port numbers. The system $\mathrm{SE}$ is obtained from averaging the SE of all users. The blanket transmission in [7] with equal PA (EPA) is illustrated for comparisons. In this method, each RRH port equally allocates its power to each subcarrier. The subcarrier is assigned to users randomly. As the number of RRH port increases, the SE improves for all the methods. On one hand, the gain comes from the diversity order brought by more transmit antennas. On the other hand, this is because the RRH ports are distributed in the area, which can shorten the transmission distance. The gap between the two proposed algorithms are rather small. This indicates that applying the PPA captures the essence of achieving the SE optimality in joint resource allocations in OFDMA-RRH.

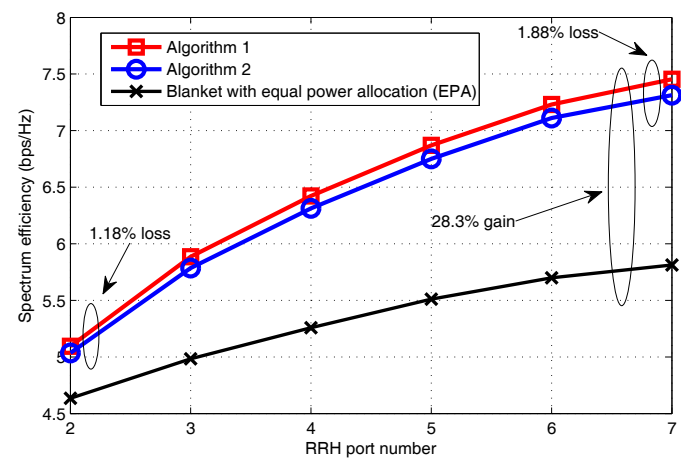

Figure 5 Spectrum efficiency (SE) performances. Figure 5 shows the spectrum efficiency $(\mathrm{bps} / \mathrm{Hz})$ of proposed algorithms and blanket transmission with equal power allocation (EPA) versus different RRH port numbers. Legend of curves from top to bottom: Algorithm 1; Algorithm 2; Blanket with equal power allocation (EPA).
In Figure 6 the power consumption is shown. It is seen that Algorithm 1 and blanket transmission always spend all the system power. In Algorithm 2, as the number of RRH ports increases, the total power consumption is decreasing. When $M=7$, approximately $8 \%$ of the system power is saved, at the cost of $1.88 \%$ loss of the system SE. In fact, the PPA requires that the allocated power on each RRH port for arbitrary subcarrier maintains the linear relationship with the channel gain. In RRH deployments where the antennas are distributed, the channel gain is determined mainly by the large-scale fading. If the distance between a RRH port and a user is long, the power allocated from this port to the user will be small, according to PPA. For example, if it is assumed that $m_{0}$ is quite the farthest RRH port away from user $k$, there will be a high possibility that the combined channel gain between them will be the smallest, compared with those of other RRH ports, i.e., $G_{k}\left[m_{0}, n\right] \ll G_{k}[m, n], m \neq m_{0}, \forall n$. Then from PPA, we can yield $p_{k}^{*}\left[m_{0}, n\right] \ll p_{k}^{*}[m, n], m \neq m_{0}, \forall n$, which means RRH port $m_{0}$ do not need to be sufficiently used. Therefore, if it happens that most of the users are relatively far from certain RRH port, this port may be only partially used. In this situation, its power can be saved by Algorithm 2.

To prove this description, in Figure 6 we also show the power consumption results for central antenna deployments, where Algorithm 2 is applied but all the RRH ports are located in cell center and the subcarriers are assigned to users randomly. Other simulation configurations remain the same. In this case, one user has the same large-scale fading to each RRH port. The discrepancy between the combined channel gains only relies on fluctuations of the small-scale fading. In Figure 6, it is shown that almost no power could be saved. In fact, the

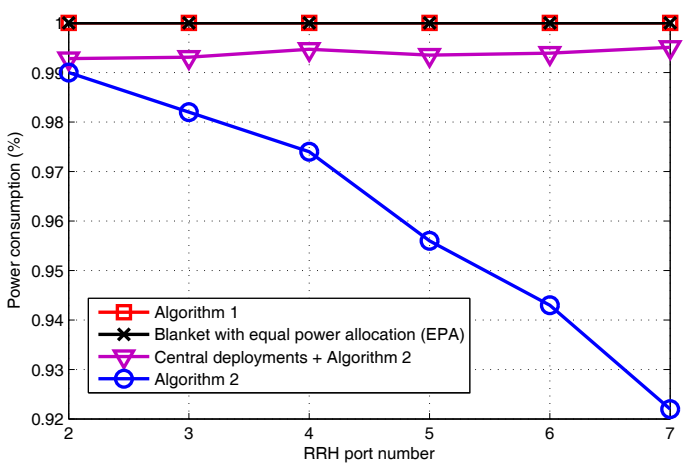

Figure 6 Power consumption comparisons. Figure 6 shows the power consumption of proposed algorithms in RRH and center deployments, and blanket transmission with equal power allocation (EPA) versus different RRH port numbers. Legend of curves from top to bottom: Algorithm 1; Blanket with equal power allocation (EPA); Central deployments + Algorithm 2; Algorithm 2. 
differences of power consumption among RRH ports are averaged over the large amount of subcarriers.

For Algorithm 1, it can be supposed that the exhausted power is divided into two parts. The first part makes the major contribution to the system SE, yet the other part only makes little improvement, just to achieve the optimal SE value from the mathematical point of view. By applying the PPA, the power is utilized in a more efficient way. This is to explore the first part of power and save the other part. Figure 7 illustrates the EE comparison results. Among them, thanks to the power-saving, Algorithm 2 is shown to have the best $\mathrm{EE}$ performance.

Taken $\boldsymbol{\lambda}$ as an example, Figure 8 shows the snapshots of the iteration to update $\lambda$ in Algorithm 1 for four random channel realizations when $M=7$. Other variables and Lagrange multipliers in Algorithm 2 follow a similar trend of convergence. Frobenius norm of $\lambda$ is plotted. It is seen that an acceptable iteration number, which is less than 10, is required for convergence. This proves the stability of the proposed algorithm.

\section{Conclusion}

This article deals with the optimal and energy-saving joint resource allocations in OFDMA-RRH. From a novel view, it proposes that in optimal resource allocations, not all the power is used efficiently. By telling the efficient part from the inefficient part, considerable system power can be saved. We believe this idea can be applied to other related resource allocation problems in OFDMA-RRH, which is our on going study.

\section{Appendix 1: Proof of Lemma 1}

Since $\lambda_{m} \leq 0$, from the perspective of analytic geometry, denote

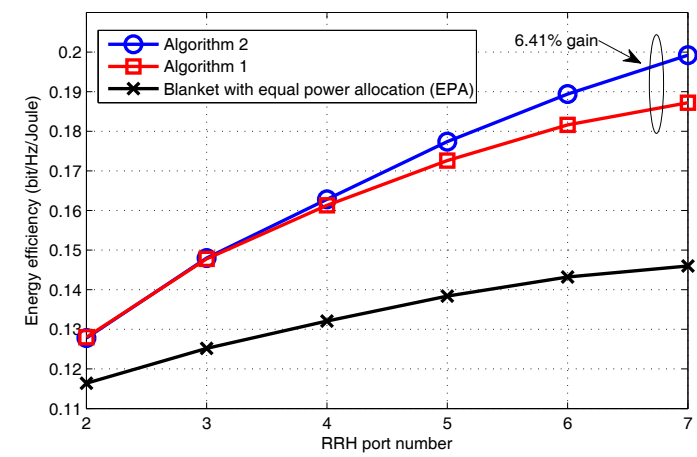

Figure 7 Energy efficiency (EE) performances. Figure 7 shows the energy efficiency (bit/Hz/Joule) of proposed algorithms and blanket transmission with equal power allocation (EPA) versus different RRH port numbers. Legend of curves from top to bottom: Algorithm 2; Algorithm 1; Blanket with equal power allocation (EPA).

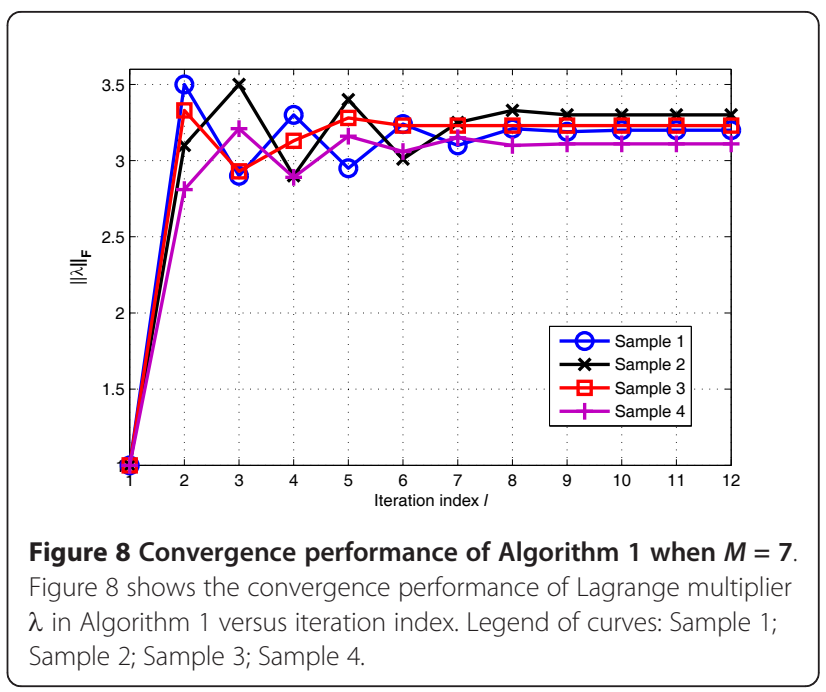

$$
q_{m}=\sqrt{-\lambda_{m} p_{k}[m, n]}, a_{m}=\frac{\sqrt{G_{k}[m, n]}}{\sigma \sqrt{-\lambda_{m}}}, m=1,2, \ldots, M .
$$

P2 can be transformed into:

$$
\text { P4 : } \max _{q} \log \left[1+\left(\sum_{m=1}^{M} a_{m} q_{m}\right)^{2}\right]-\sum_{m=1}^{M} q_{m}^{2} .
$$

In a $M$-dimensional space, assume $\boldsymbol{a}=\left(a_{1}, \ldots, a_{M}\right)^{T}$, $\boldsymbol{q}=\left(q_{1}, \ldots, q m\right)^{T}$ and $Q$ represents the point $\left(q_{1}, \ldots, q_{M}\right)$. For $\forall b \geq 0$, all the points $Q$ that satisfies $\boldsymbol{a}^{T} \boldsymbol{q}=\sum_{m=1}^{M} a_{m} q_{m}=b$ form a hyperplane $\left\{\boldsymbol{q} \mid \boldsymbol{a}^{T} \boldsymbol{q}=b\right\}$, with $\boldsymbol{a}$ as its normal vector. It is not hard to find that for $\forall Q$ in the hyperplane, the value of the first part of $\mathrm{P} 4, \log \left[1+\left(\sum_{m=1}^{M} a_{m} q_{m}\right)^{2}\right]$ is fixed. As for the second part, $\sum_{m=1}^{M} q_{m}^{2}$, it represents the square of the distance between point $Q$ and the origin of coordinates, which should be minimized. As shown in Figure 9. it is obvious that among all the points in the hyperplane $\{\boldsymbol{q} \mid$ $\left.\boldsymbol{a}^{T} \boldsymbol{q}=b\right\}$, the nearest point from the origin is the intersection point of ray $\{\theta \boldsymbol{a} \mid, \theta \geq 0\}$ and the hyperplane itself. This indicates that, in the optimal solution of $\mathrm{P} 4$, vector $\boldsymbol{q}$ should be along with the direction of $\boldsymbol{a}$, i.e., $\boldsymbol{q}$ and $\boldsymbol{a}$ are linearly dependent.

Let $\boldsymbol{q}=u \boldsymbol{a}$, then $\mathrm{P} 4$ can be transformed into:

$$
\max _{u} \log \left[1+\left(\sum_{m=1}^{M} u a_{m}^{2}\right)^{2}\right]-\sum_{m=1}^{M} u^{2} a_{m}^{2} .
$$

Define $f(u)=\log \left(1+A^{2} u^{2}\right)-A u^{2}$, where $A=\sum_{m=1}^{M} a_{m}^{2}$. We have: 


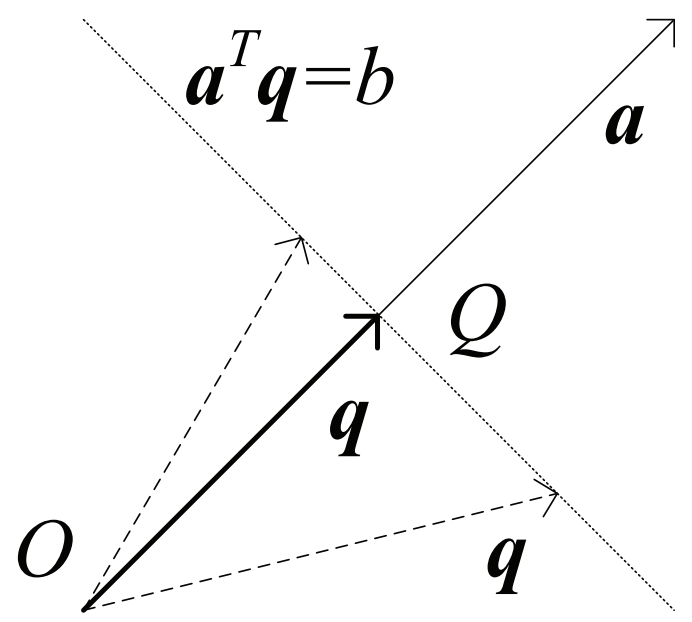

Figure 9 Direction of vector $q$ for the optimality. Figure 9 shows the relationship between $\boldsymbol{q}$ and $\boldsymbol{a}$ to achieve the optimality for P4 in a M-dimensional space.

$$
\frac{\partial f(u)}{\partial u}=\frac{2 A^{2} u}{\ln 2\left(1+A^{2} u^{2}\right)}-2 A u=0 .
$$

The optimal solution is $u^{*}=\frac{\sqrt{|A / \ln 2-1|}}{A}$. From $\boldsymbol{q}=$ $u^{*} \boldsymbol{a}$ and Equation (31), $p_{k}^{*}[m, n]$ is given by:

$$
p_{k}^{*}[m, n]=\left[\frac{B-\ln 2}{B^{2} \ln 2} \cdot \frac{G_{k}[m, n]}{\sigma^{2} \lambda_{m}^{2}}\right]^{+},
$$

where $B=\sum_{m=1}^{M} \frac{G_{k}[m, n]}{-\sigma^{2} \lambda_{m}}$.

We should note that $p_{k}^{*}[m, n]$ always has a positive value. In fact, according to LTE configurations [4], it simply satisfies that $\frac{G_{k}[m, n]}{\sigma^{2}} \gg 1$ in the simulation. The initial value of $\lambda_{m}\left(\lambda_{m} \leq 0\right)$ is generated according to $\left\|\lambda_{m}\right\|_{F}=1$ and will be a small number in the iteration, as shown in Figure 8. Therefore, we have $\sum_{m=1}^{M} \frac{G_{k}[m, n]}{-\sigma^{2} \lambda_{m}}>\ln 2$ and $p_{k}^{*}[m, n]>0$.

\section{Appendix 2: Proof of Lemma 2}

According to Equation (23), for $\forall \lambda^{\prime}$, we have

$$
g\left(\boldsymbol{\lambda}^{\prime}\right)=\max _{\boldsymbol{p}, \boldsymbol{\rho}} \mathcal{L}\left(\boldsymbol{p}\left(\boldsymbol{\lambda}^{\prime}\right), \rho\left(\boldsymbol{\lambda}^{\prime}\right), \boldsymbol{\lambda}^{\prime}\right),
$$

where

$$
\begin{aligned}
\mathcal{L}\left(p\left(\lambda^{\prime}\right), \rho\left(\lambda^{\prime}\right), \lambda^{\prime}\right)= & \sum_{k=1}^{K} \sum_{n=1}^{N} \rho_{k}[n]\left(\lambda^{\prime}\right) \cdot \log \left(1+\frac{1}{\sigma^{2}}\left(\sum_{m=1}^{M} \sqrt{G_{k}[m, n] p_{k}[m, n]\left[\lambda^{\prime}\right)}\right)^{2}\right) \\
& +\sum_{j=1}^{M} \lambda_{j}^{\prime}\left(\sum_{k=1}^{K} \sum_{n=1}^{N} \rho_{k}[n]\left[\lambda^{\prime}\right) p_{k}[m, n]\left[\lambda^{\prime}\right)-P\right) .
\end{aligned}
$$

Let $\rho^{*}(\lambda), \boldsymbol{p}^{*}(\lambda)$ be the optimal solution for minimizing $g(\lambda)$, according to Equation (37), we have:

$$
\begin{aligned}
g\left(\lambda^{\prime}\right) \geq & \sum_{k=1}^{K} \sum_{n=1}^{N} \rho_{k}^{*}[n](\lambda) \cdot \log \left(1+\frac{1}{\sigma^{2}}\left(\sum_{m=1}^{M} \sqrt{G_{k}[m, n] p_{k}^{*}[m, n](\lambda)}\right)^{2}\right) \\
& +\sum_{j=1}^{M} \lambda_{j}^{\prime}\left(\sum_{k=1}^{K} \sum_{n=1}^{N} \rho_{k}^{*}[n](\lambda) p_{k}^{*}[m, n](\lambda)-P\right) .
\end{aligned}
$$

The right-hand side of the above inequality can be rewritten as

$$
g\left(\lambda^{\prime}\right) \geq g(\lambda)+\sum_{j=1}^{M}\left(\lambda_{j}^{\prime}-\lambda_{j}\right)\left(\sum_{k=1}^{K} \sum_{n=1}^{N} \rho_{k}^{*}(\lambda) p_{k}^{*}[m, n](\lambda)-P\right),
$$

which verifies the definition of sub-gradient and completes the proof.

\section{Appendix 3: Proof of Lemma 3}

The derivative of $f(x)$ is

$$
f(x)^{\prime}=\frac{1}{x^{2}}\left[\frac{w x}{(1+w x) \ln 2}-\log (1+w x)\right],
$$

where $\frac{1}{x^{2}}$ is positive if $x>0$ and denote

$$
g(x)=\frac{w x}{(1+w x) \ln 2}-\log (1+w x) .
$$

The derivative of $g(x)$ is

$$
g(x)^{\prime}=\frac{-w^{2} x}{(1+w x)^{2} \ln 2}<0, \quad x>0,
$$

which indicates that $g(x)$ is monotonically decreasing when $x>0$. Since $g(0)=0$, it follows $g(x)<0$, when $x>$ 0 . This proves that $f(x)^{\prime}<0$ from Equation (40). Therefore, $f(x)$ is monotonically decreasing when $x>0$.

\section{Appendix 4: Proof of Lemma 4}

The problem could be formulated as:

$$
\begin{aligned}
\max _{p_{k}[m, n]} & \frac{1}{\sigma^{2}}\left(\sum_{m=1}^{M} \sqrt{G_{k}[m, n]} \sqrt{p_{k}[m, n]}\right)^{2}, \\
\text { s.t. } & \sum_{m=1}^{M} p_{k}[m, n]=\mu_{k}[n] P_{T}, \quad p_{k}[m, n] \geq 0, \quad \forall m, n .
\end{aligned}
$$

From the Cauchy-Schwarz inequality, it satisfies:

$$
\begin{aligned}
\frac{1}{\sigma^{2}}\left(\sum_{m=1}^{M} \sqrt{G_{k}[m, n]} \sqrt{p_{k}[m, n]}\right)^{2} & \leq \frac{1}{\sigma^{2}} \sum_{m=1}^{M}\left(\sqrt{\sigma_{k}[m, n]}\right)^{2} \sum_{m=1}^{M}\left(\sqrt{p_{k}[m, n]}\right)^{2} \\
& \left.=\frac{1}{\sigma^{2}} \sum_{m=1}^{M} G_{k}[m, n] \sum_{m=1}^{M} p_{k}[m, n]=\frac{1}{\sigma^{2}} \mu_{k} \mid n\right] P_{T} \cdot \sum_{m=1}^{M} G_{k}[m, n] .
\end{aligned}
$$

The first inequality in (44) holds if and only if $\sqrt{G_{k}[m, n]}$ and $\sqrt{p_{k}[m, n]}$ are linearly dependent, which is:

$$
p_{k}^{*}[m, n]=\frac{G_{k}[m, n]}{\sum_{m=1}^{M} G_{k}[m, n]} \cdot \mu_{k}[n] P_{T} .
$$




\section{Appendix 5: Proof of Lemma 5 \\ Proof of 5.1:}

When $\boldsymbol{p}_{k}^{*}[n]$ is linearly increased to $\boldsymbol{p}_{k}^{\prime}[n]$, according to Lemma 4 we can yield:

$$
\gamma_{k}^{*}[n]=\frac{1}{\sigma^{2}} \sum_{m=1}^{M} G_{k}[m, n] \sum_{m=1}^{M} p_{k}^{*}[m, n] \leq \frac{1}{\sigma^{2}} \sum_{m=1}^{M} G_{k}[m, n] \sum_{m=1}^{M} p_{k}^{\prime}[m, n]=\gamma_{k}^{\prime}[n] .
$$

Rewrite $\eta$ from Equation (17) as:

$$
\eta(P)=\frac{1}{P} \log _{2}(1+\gamma)=\frac{1}{P} \log _{2}\left(1+\frac{1}{\sigma^{2}} P \sum_{m=1}^{M} G_{k}[m, n]\right) .
$$

Substituting $\gamma_{k}^{*}[n]$ and $\gamma_{k}^{\prime}[n]$ into Equation (47), from Lemma 3, it is easy to find out that:

$$
\begin{aligned}
\eta\left(\sum_{m=1}^{M} p_{k}^{*}[m, n]\right) & =\frac{1}{\sum_{m=1}^{M} p_{k}^{*}[m, n]} \log _{2}\left(1+\frac{1}{\sigma^{2}} \sum_{m=1}^{M} G_{k}[m, n] \sum_{m=1}^{M} p_{k}^{*}[m, n]\right) \\
& \geq \frac{1}{\sum_{m=1}^{M} p_{k}^{\prime}[m, n]} \log _{2}\left(1+\frac{1}{\sigma^{2}} \sum_{m=1}^{M} G_{k}[m, n] \sum_{m=1}^{M} p_{k}^{\prime}[m, n]\right)=\eta\left(\sum_{m=1}^{M} p_{k}^{\prime}[m, n]\right),
\end{aligned}
$$

which shows the reduction of EE.

\section{Proof of 5.2:}

Besides $p_{k}^{*}[1, n]=P_{0}$, let the PA for other RRH ports, $p_{k}^{*}[i, n]$, increase to $p_{k}^{\prime \prime}[i, n], i=2, \ldots, M$ (PCPP should be guaranteed for these RRH ports), and the related SNR is represented as $\gamma_{k}^{\prime \prime}[n]$. The sum amount of the increased PA will be:

$$
\hat{P}_{k}[n]=\sum_{m=1}^{M} p_{k}^{\prime \prime}[m, n]-\sum_{m=1}^{M} p_{k}^{*}[m, n] .
$$

To make comparisons, first it is assumed that $\hat{P}_{k}[n]$ is proportionally allocated to all the RRH ports according to Lemma 4 with the optimal SNR $\hat{\gamma}_{k}[n]$. From Lemma 5.1 and Equation (47), we will have:

$$
\eta\left(\sum_{m=1}^{M} p_{k}^{*}[m, n]\right) \geq \eta\left(\sum_{m=1}^{M} p_{k}^{*}[m, n]+\hat{P}_{k}[n]\right)=\eta\left(\sum_{m=1}^{M} p_{k}^{\prime \prime}[m, n]\right) .
$$

Second, since Lemma 4 gives the best SNR for a given total power $\sum_{m=1}^{M} p_{k}^{\prime \prime}[m, n]$, i.e., $\hat{\gamma}_{k}[n] \geq \gamma_{k}^{\prime \prime}[n]$, there is:

$$
\begin{aligned}
\eta\left(\sum_{m=1}^{M} p_{k}^{\prime \prime}[m, n]\right) & =\frac{1}{\sum_{m=1}^{M} p_{k}^{\prime \prime}[m, n]} \log _{2}\left(1+\hat{\gamma}_{k}[n]\right) \\
& \geq \frac{1}{\sum_{m=1}^{M} p_{k}^{\prime \prime}[m, n]} \log _{2}\left(1+\gamma_{k}^{\prime \prime}[n]\right)=\eta^{\prime}\left(\sum_{m=1}^{M} p_{k}^{\prime \prime}[m, n]\right) .
\end{aligned}
$$

From both (50) and (51), we have $\eta\left(\sum_{m=1}^{M} p_{k}^{*}[m, n]\right) \geq \eta^{\prime}\left(\sum_{m=1}^{M} p_{k}^{\prime \prime}[m, n]\right)$, which completes the proof.

\section{Proof of 5.3:}

Lemma 5.3 can be proved similarly as 5.1 . We omit the process to avoid duplication.

\section{Acknowledgements}

This study was supported by Project NSF61121001, National Key Basic Research Program of China (973 Program) 2009CB320406, Chinese National Key Project under Grant No. 2010ZX03002-003-03, Chinese Program for New Century Excellent Talents in University (NCET) and EU FP7 S2EuNet project (247083)

\section{Author details}

'Key Laboratory of Universal Wireless Communication, Ministry of Education, Beijing, China ${ }^{2}$ Wireless Technology Innovation Institute, Beijing University of Posts and Telecommunications, Beijing, China

Received: 20 July 2011 Accepted: 15 March 2012

Published: 15 March 2012

\section{References}

1. H Bolcskei, D Gesbert, C Papadias, AJ van der Veen, Space-Time Wireless Systems: From Array Processing to MIMO Communications (Cambridge University Press, Cambridge, 2006)

2. C Wong, R Cheng, K Lataief, R Murch, Multiuser OFDM with adaptive subcarrier, bit, and power allocation. IEEE J Sel Areas Commun. 17(10), 1747-1758 (1999). doi:10.1109/49.793310

3. E Oh, B Krishnamachari, X Liu, Z Niu, Towards dynamic energy-efficient operation of cellular network infrastructure. IEEE Commun Mag. 49(6), 56-61 (2011)

4. $\quad 3 G P P,\{T R$ 36.814 V9.0.0: Further Advancements for E-UTRA, Physical Layer Aspects\}. (2010)

5. S Zhou, M Zhao, X Xu, J Wang, Y Yao, Distributed wireless communication system: a new architecture for future public wireless access. IEEE Commun Mag. 41(3), 108-113 (2003). doi:10.1109/MCOM.2003.1186553

6. W Roh, A Paulraj, MIMO channel capacity for the distributed antenna, in IEEE 56th Proceedings on Vehicular Technology Conference, vol. 2. (Vancouver, BC, Sep, 2002), pp. 706-709

7. W Choi, J Andrews, Downlink performance and capacity of distributed antenna systems in a multicell environment. IEEE Trans Wirel Commun. 6 , 69-73 (2007)

8. N Dawod, I Marsland, R Hafez, Improved transmit steering for MIMO-OFDM downlinks with distributed base station antenna arrays. IEEE J Sel Areas Commun. 24(3), 419-426 (2006)

9. J Zhang, R Chen, J Andrews, A Ghosh, R Heath, Networked MIMO with clustered linear precoding. IEEE Trans Wirel Commun. 8(4), 1910-1921 (2009)

10. T Koike-Akino, A Molisch, C Duan, Z Tao, P Orlik, Capacity, MSE and secrecy analysis of linear block precoding for distributed antenna systems in multiuser frequency-selective fading channels. IEEE Trans Commun. 59(3), 888-900 (2011)

11. $X$ Wang, $P$ Zhu, M Chen, Antenna location design for generalized distributed antenna systems. IEEE Commun Lett. 13(5)، 315-317 (2009)

12. P Marsch, G Fettweis, A decentralized optimization approach to backhaulconstrained distributed antenna systems, in IEEE 16th IST, Mobile and Wireless Communications Summit, Budapest, 1-5 (July 2007)

13. H Zhang, N Mehta, A Molisch, J Zhang, H Dai, Asynchronous interference mitigation in cooperative base station systems. IEEE Trans Wirel Commun. 7, 155-165 (2008)

14. M Karakayali, G Foschini, R Valenzuela, Network coordination for spectrally efficient communications in cellular systems. IEEE Wirel Commun. 13(4), 56-61 (2006). doi:10.1109/MWC.2006.1678166

15. W Yu, T Lan, Transmitter optimization for the multi-antenna downlink with per-antenna power constraints. IEEE Trans Signal Process. 55(6), 2646-2660 (2007)

16. S Xinghua, $\mathrm{H}$ Zhiqiang, $\mathrm{N}$ Kai, W Weiling, A hierarchical resource allocation for OFDMA distributed wireless communication systems, in IEEE Global Telecommunications Conference 2007, Washington, DC, pp. 5195-5199 (Nov 2007)

17. K Lee, $\mathrm{V}$ Leung, Fair allocation of subcarrier and power in an OFDMA wireless mesh network. IEEE J Sel Areas Commun. 24(11), 2051-2060 (2006)

18. L Ling, T Wang, Y Wang, C Shi, Schemes of power allocation and antenna port selection in OFDM distributed antenna systems, in IEEE 72nd Vehicular Technology Conference Fall, Ottawa, ON, pp. 1-5 (Sept. 2010) 
19. P Gong, K Yu, Y Wang, Radio resource allocation for multiuser OFDMA distributed antenna systems, in IEEE International Conference on Network Infrastructure and Digital Content 2009, Beijing, pp. 912-916 (Nov. 2009)

20. F Meshkati, H Poor, S Schwartz, N Mandayam, An energy-efficient approach to power control and receiver design in wireless data networks. IEEE Trans Commun. 53(11), 1885-1894 (2005). doi:10.1109/TCOMM.2005.858695

21. G Miao, N Himayat, Y Li, D Bormann, Energy efficient design in wireless OFDMA, in IEEE International Conference on Communications 2008, Beijing, pp. 3307-3312 (May 2008)

22. B Badic, T O'Farrrell, P Loskot, J He, Energy efficient radio access architectures for green radio: large versus small cell size deployment, in IEEE 70th Vehicular Technology Conference Fall, Anchorage, AK, pp. 1-5 (Sept. 2009)

23. F Richter, A Fehske, G Fettweis, Energy efficiency aspects of base station deployment strategies for cellular networks, in IEEE 70th Vehicular Technology Conference Fall, Anchorage, AK, pp. 1-5 (Sept. 2009)

24. W Yu, R Lui, Dual methods for nonconvex spectrum optimization of multicarrier systems. IEEE Trans Commun. 54(7), 1310-1322 (2006)

25. S Boyd, L Vandenberghe, Convex optimization, (Cambridge University Press, Cambridge, 2004)

26. G Li, Fundamental trade-offs on Green wireless networks. IEEE Commun Mag 31 (2011)

doi:10.1186/1687-1499-2012-111

Cite this article as: Wang et al:: Joint resource allocations for remote radio head deployments with coherent transmitter. EURASIP Journal on Wireless Communications and Networking 2012 2012:111.

\section{Submit your manuscript to a SpringerOpen ${ }^{\mathcal{O}}$ journal and benefit from:}

- Convenient online submission

- Rigorous peer review

- Immediate publication on acceptance

- Open access: articles freely available online

- High visibility within the field

- Retaining the copyright to your article

Submit your next manuscript at $\gg$ springeropen.com 